\title{
VITRECTOMÍA ENZIMÁTICA POR INYECCIÓN INTRAVÍTREA DE PLASMINA AUTÓLOGA COMO TRATAMIENTO INICIAL DE LAS MEMBRANAS EPIRRETINIANAS MACULARES Y EL SÍNDROME DE TRACCIÓN VITREOMACULAR
}

\section{ENZYMATIC VITRECTOMY BY INTRAVITREAL AUTOLOGOUS PLASMIN INJECTION AS INITIAL TREATMENT FOR MACULAR EPIRETINAL MEMBRANES AND VITREOMACULAR TRACTION SYNDROME}

\author{
DÍAZ-LLOPIS M ${ }^{1}$, UDAONDO P ${ }^{2}$, CERVERA E ${ }^{3}$, GARCÍA-DELPECH S ${ }^{4}$, SALOM D ${ }^{2}$, QUIJADA A ${ }^{5}$, \\ ROMERO FJ ${ }^{6}$
}

\begin{abstract}
RESUMEN
Objetivo: Determinar si la inyección intravítrea de plasmina es efectiva en el tratamiento de las membranas epirretinianas maculares (MEM) y el síndrome de tracción vitreomacular (STVM), sin necesidad de asociar vitrectomía quirúrgica.

Material y método

Diseño: Estudio piloto prospectivo, de intervención, serie de casos.

Pacientes: Siete pacientes, cuatro con membrana epirretiniana macular y tres con síndrome de tracción vitreomacular.
\end{abstract}

\begin{abstract}
Purpose: To determine the effectiveness of intravitreal plasmin injection in the treatment of macular epiretinal membranes (MEM) and vitreomacular traction syndrome (VMTS) without associated pars plana vitrectomy. Methods:

Design: Interventional, prospective, case series pilot study.

Patients: Seven patients were enrolled in the study, 4 with MEM and 3 with VMTS.

Treatment: $0.2 \mathrm{ml}$ of autologous plasmin intravitreally injected under topical anesthesia was administered
\end{abstract}

\footnotetext{
Recibido: 19/5/08. Aceptado: 12/1/09.

${ }^{1}$ Departamento de Oftalmología. Hospital La Fe de Valencia. Valencia. España. Unidad Docente de Oftalmología. Universidad de Valencia.

España. Universidad Católica San Vicente Ferrer. Valencia. España.

2 Departamento de Oftalmología. Hospital La Fe de Valencia. Valencia. España.

3 Departamento de Oftalmología. Hospital General Universitario de Valencia. España.

4 Departamento de Oftalmología. Hospital General Universitario de Valencia. España. Universidad Católica de Valencia. Valencia. España.

5 Universidad de Valencia. Valencia. España.

${ }^{6}$ Universidad CEU-San Pablo. Valencia. España. Fundación Oftalmológica del Mediterráneo (FOM). Valencia. España.
}

Correspondencia:

Manuel Díaz Llopis

Cl. Ruzafa, 19

46004 Valencia

España

E-mail: manuel.diaz@uv.es 
Tratamiento: Inyección intravítrea de $0,2 \mathrm{ml}$ de plasmina autóloga bajo anestesia tópica. La plasmina fue obtenida por un método simplificado modificado por adicción de uroquinasa.

Principales medidas: Grado de separación de la membrana epirretiniana de la retina medido por tomografía de coherencia óptica (OCT) y la mejor agudeza visual corregida (escala Snellen), antes y al mes de la inyección de plasmina.

Resultados: Todos los pacientes completaron el seguimiento de 1 mes. La membrana epirretiniana (MEM) permaneció oftalmoscópicamente adherida, sin cambios ni separación del plano de la retina. Sin embargo, en los casos con síndrome de tracción vitreomacular (STVM), la membrana se despegó del área foveal, desapareciendo los pliegues retinianos traccionales secundarios, y normalizando la mácula la estructura anatómica. La agudeza visual no se modificó en ninguno de los casos de membrana epirretiniana y mejoró en los casos de síndrome de tracción vitreomacular. No se observaron efectos secundarios.

Conclusión: La inyección intravítrea de plasmina autóloga como tratamiento inicial previo a la vitrectomía quirúrgica, en la serie de casos estudiada, parece ineficaz en las membranas epirretinianas maculares, y más eficaz en el síndrome de tracción vitreomacular, mejorando en este último la agudeza visual y consiguiendo el despegamiento al menos parcial de la membrana. Estudios más amplios son necesarios para confirmar su eficacia y la posibilidad de incrementar la misma con reinyecciones periódicas de plasmina.

Palabras clave: Plasmina autóloga, vitreolisis farmacológica, membrana epirretiniana, síndrome de tracción vitreomacular, vitrectomía enzimática, vitreolisis enzimática. to all patients. The plasmin was obtained by a simplified method with urokinase.

Main outcome measures: Degree of detachment of the MEM and the VMTS measured by optical coherence tomography (OCT), and the best corrected visual acuity (Snellen scale) before and one month after the plasmin injection.

Results: The follow-up period was completed by all the patients. The MEM remained attached to the retina in all cases, as measured either by biomicroscopy or OCT. The VMTS was completely detached from the foveal area in all cases, with the disappearance of secondary tractional retinal folds and recovery of the normal macular anatomic architecture as measured by OCT. Visual acuity was not modified in any of the MEM patients, and improved in all VMTS patients. No adverse effects were observed. Conclusion: In our case series, intravitreally injected autologous plasmin was not effective in the treatment of MEM, but resolved VMTS successfully, improving the visual acuity and releasing the retinal traction without the need for associated pars plana vitrectomy. Larger studies to confirm the efficacy of this technique and the possibility of success after repeated injections are warranted (Arch Soc Esp Oftalmol 2009; 84: 91-100).

Key words: Autologous plasmin, pharmacologic vitreolysis, epiretinal membrane, macular pucker, vitreomacular traction syndrome, enzimatic vitrectomy, enzymatic vitreolysis.

\section{INTRODUCCIÓN}

La formación de membranas vitreorretinianas y epirretinianas, y una adhesión patológica vitreomacular, son signos característicos tanto de la vitreorretinopatía proliferante como de la fibrosis prerretiniana macular («macular pucker»), el síndrome de tracción vitreomacular y los agujeros maculares (13). La matriz extracelular de las membranas vitreorretinianas y epirretinianas contiene abundante fibronectina y laminina $(4,5)$, y están en permanente formación y degradación biológica. La cascada proteolítica mediada por activación del plasminógeno, a través de la formación de plasmina, es un importante mecanismo proteolítico de la degradación fisiológica en la dinámica permanente de remodelación tisular de la matriz extracelular de dichas membranas (6).

Se han descrito ocasionalmente casos de separación espontánea de las membranas epirretinianas maculares, 
en el contexto de un desprendimiento de vítreo posterior $(7,8)$, y hasta en el $11 \%$ de los casos de síndrome de tracción vitreomacular, con mejoría inmediata de la agudeza visual y desaparición de las metamorfopsias (9-14). Además, la presencia de un desprendimiento de vítreo posterior facilita el pelado quirúrgico durante la vitrectomía de las membranas $(15,16)$.

La vitreolisis farmacológica ha sido propuesta por diversos autores (16-20). La plasmina, es una proteasa con acción enzimática sobre la laminina y la fibronectina (21-25), que están localizadas entre el cortex vítreo posterior y la membrana limitante interna de la retina, y se consideran las principales moléculas implicadas como responsables de la firme adhesión entre ambas superficies $(26,27)$. Diversos estudios han descrito la eficacia de la inyección de plasmina, inmediatamente previa a la vitrectomía, en casos de retinopatía diabética proliferante y/o con edema macular refractario, agujeros maculares y retinopatía del prematuro (28-34), simplificando la cirugía al facilitar el despegamiento quirúrgico de la hialoides posterior y de las membranas vitreorretinianas (15).

La inyección intravítrea de plasmina -sin vitrectomía quirúrgica asociada-, como tratamiento único, que ha sido bautizada como vitrectomía farmacológica y enzimática $(16,18)$, ha demostrado su eficacia en el tratamiento del edema macular diabético difuso tanto como tratamiento inicial como en los casos refractarios a otros tratamientos, al inducir un desprendimiento posterior del vítreo (DPV) enzimáticamente (35) (Díaz-Llopis M, Udaondo P, García-Delpech S, Salom D, Cervera E, Quijada A. Intravitreal autologous plasmin without associated vitrectomy for refractory diffuse diabetic macular edema. Eye 2008; in press).

El objetivo del presente estudio piloto, sobre una serie de pacientes con membrana epirretiniana macular (MEM) y síndrome de tracción vitreomacular (STVM), es establecer la eficacia de la inyección autóloga de plasmina -sin asociar vitrectomía-, como única e inicial opción terapéutica en el tratamiento.

\section{SUJETOS, MATERIAL Y MÉTODO}

Este estudio es un estudio piloto prospectivo y de intervención terapéutica. Los pacientes fueron reclutados entre septiembre 2006 y diciembre de 2007 en la Unidades de Mácula de los Servicios de
Oftalmología, contando con la aprobación de los Comités de Investigación. Siete ojos de siete pacientes diferentes consecutivos fueron incluidos en el estudio para tratamiento con plasmina intravítrea. Todos los pacientes firmaron consentimiento informado, explicándoles claramente el objetivo del estudio y los potenciales riesgos de la inyección intravítrea (desprendimiento de retina, endoftalmitis, hemorragia vítrea, traumatismo del cristalino, etc) y la posibilidad de requerir tratamientos adicionales posteriores (vitrectomía quirúrgica, tinción y pelado mecánico de su membrana).

\section{Pacientes: criterios de inclusión y exclusión}

\section{Criterios de inclusión}

Sólo se incluyeron pacientes que tuvieran confirmación de su membrana epirretiniana macular o de su síndrome de tracción vitreomacular por tomografía de coherencia óptica (Stratus OCT-3, Zeiss). Sólo se incluyeron STVM y/o MEM primarias o idiopáticas, sin relación con ninguna otra patología ocular previa (retinopatía diabética, uveítis, desprendimiento de retina, etc.), estabilizadas anatómica y visualmente durante los tres meses previos a la inyección de plasmina.

\section{Criterios de exclusión}

1. Cualquier patología ocular concomitante (glaucoma, retinopatía diabética, etc).

2. Cirugía ocular previa en los seis meses previos.

3. Agudeza visual superior a 0,4 (escala Snellen).

\section{Examen preoperatorio}

Todos los pacientes recibieron una exploración oftalmológica completa antes de la inyección de plasmina, incluyendo exploración a la lámpara hendidura, tonometría de aplanación, oftalmoscopía indirecta, escaner macular por tomografía de coherencia óptica (OCT), fotografía de fondo del área macular y angiografía fluoresceínica. La tomografía de coherencia y la biomicroscopía confirmaron la adherencia de la membrana en el área macular en todos los casos, además de permitir diferenciar claramente las membranas epirretinianas del síndrome de tracción vitreomacular. 


\section{Preparación de la plasmina autóloga}

La plasmina fue preparada en el quirófano inmediatamente antes de la inyección, como ha sido descrita previamente $(15,35)$, con la modificación de sustituir como activador del plasminógeno la estreptoquinasa por la uroquinasa (36-40). Una muestra de sangre del propio paciente se extrajo de una vena periférica. Una vez centrifugada la sangre a 4,000 rpm durante 15 minutos, el plasma fue transferido a un vial con uroquinasa, que había sido incubado previamente durante 15 minutos a $37^{\circ} \mathrm{C}$. La uroquinasa fue mezclada con el plasma agitando el vial vigorosamente durante cinco minutos. La solución volvió a ser incubada durante 10 minutos más a $37^{\circ} \mathrm{C}$. Finalmente, la solución fue esterilizada a través de un filtro milipore de $0,22 \mathrm{~mm}$, y ya estaba lista para ser inyectada.

\section{Técnica de inyección}

Antes de la inyección, se realizó anestesia tópica con colirio de tetracaína al $1 \%$ al menos 3 veces y lavado conjuntival con solución de povidona. Paracentesis de cámara anterior con aguja de 27 G, para evitar el reflujo posterior al realizar la inyección intravítrea dado el volumen a inyectar. A continuación se realizó inyección intravítrea de $0,2 \mathrm{ml}$ de la solución de plasmina autóloga con aguja de 30 G, a 3,5 ó $4 \mathrm{~mm}$ del limbo dependiendo de que el paciente fuera pseudofáquico o fáquico respectivamente. Aplicación de hemosteta en el punto de inyección para evitar el reflujo. Colirio de ciprofloxacino y dexametasona 4 veces día durante los cinco días postoperatorios. Una única inyección de plasmina por ojo fue realizada independientemente del grado de respuesta inicial.

\section{Principales medidas}

Las principales medidas del grado de eficacia y de respuesta al tratamiento fueron los cambios en la agudeza visual y la separación de la membrana de la retina. Para evitar sesgos y diferencias interobservador, todas las agudezas visuales (optotipos Snellen) y las mediciones en la tomografía de coherencia (OCT-3, Zeiss) fueron medidas por un único explorador que desconocía los datos clínicos de los pacientes (SGD). Las mediciones se realizaron el día previo, a los 7 días -datos no mostrados- y 4 semanas de la inyección. La serie de casos no permitían realizar estudio estadístico.

\section{RESULTADOS}

Siete ojos fueron incluidos en el estudio procedentes de un total de siete pacientes diferentes. La edad media de los pacientes era de 66 años (rango 42-81). Cuatro eran mujeres y tres hombres. Tres eran pseudofáquicos y cuatro fáquicos. Se inyectó plasmina en tres ojos derechos y cuatro ojos izquierdos. Todos los pacientes tenían una membrana epirretiniana macular o un síndrome de tracción vitreomacular como única patología ocular (tabla I).

\section{Agudeza visual}

No existieron cambios en la agudeza visual de ninguno de los pacientes con MEM $(0,05,0,1,0,2$, $0,3)$. En los tres pacientes con STVM mejoró la agudeza visual desde 0,1 a $0,6,0,4$ a 0,9 y de 0,2 a 0,8 respectivamente.

\section{Separación de la membrana de la retina}

No hubo cambios ni oftalmoscópicos ni en la tomografía OCT de los cuatro pacientes con MEM (figs. 1 y 2), y sí en los tres con STVM donde se observó una clara tendencia a la normalización de la estructura macular (figs. 3 y 4).

\section{Efectos secundarios}

No se observaron efectos secundarios durante el seguimiento tipo catarata, vitreítis, endoftalmitis, hemorragia vítrea, desgarro retiniano o desprendimiento de retina.

\section{DISCUSIÓN}

Los resultados de este estudio piloto y prospectivo muestran la eficacia de la inyección intravítrea de plasmina en mejorar la agudeza visual y reducir el engrosamiento macular retiniano en los pacientes con síndrome de tracción vitreomacular (STVM), y 
Tabla I. Características clínicas de los pacientes con síndrome de tracción vitreomacular y membrana epirretiniana, agudeza visual antes y 1 mes después de la inyección intravítrea de plasmina autóloga, sin asociar vitrectomía

\begin{tabular}{lcccc}
\hline N. ${ }^{\circ}$ Paciente & Edad & Diagnóstico & AV Preinyección** & AV Postinyección** \\
\hline 1 & 72 & MEM & 0,1 & 0,1 \\
2 & 67 & MEM & 0,05 & 0,05 \\
3 & 52 & MEM & 0,3 & 0,3 \\
4 & MEM & 0,2 & 0,2 \\
5 & 86 & STVM & 0,1 & 0,6 \\
6 & 41 & STVM & 0,4 & 0,9 \\
7 & 70 & STVM & 0,2 & 0,8 \\
\hline
\end{tabular}

AV: agudeza visual; **: Pre y post inyección intravítrea de plasmina autóloga; STVM: síndrome de tracción vitreomacular; MEM: membrana epirretiniana.

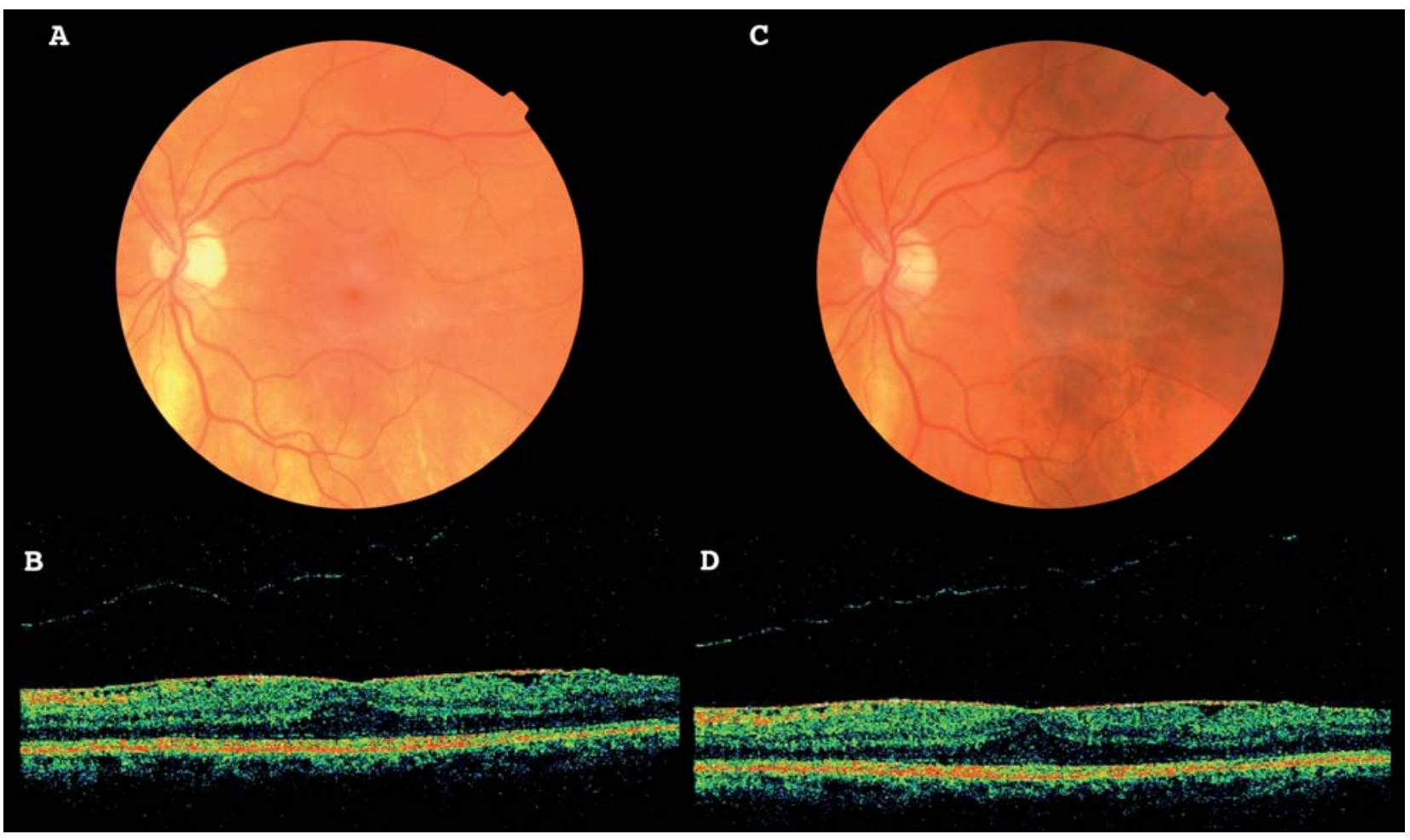

Fig. 1: Membrana epirretiniana (MEM) antes $\left(\begin{array}{l}A \\ \text { y }\end{array}\right.$ B) y después $(C$ y D $)$ de la inyección intravítrea de plasmina autóloga (paciente 3). Sin cambios anatómicos ni visuales.

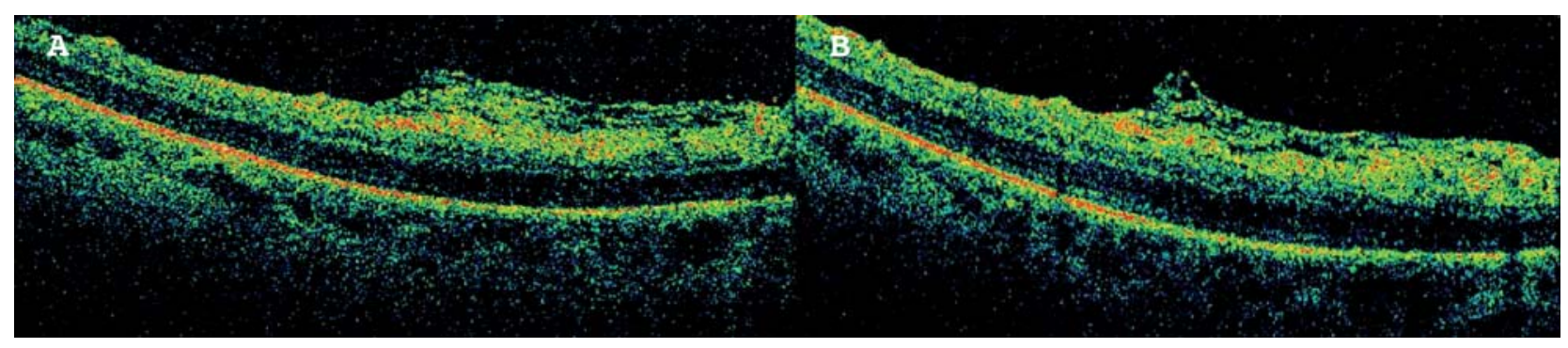

Fig. 2: Tomografía de coherencia óptica de membrana epirretiniana (MEM) antes (A) y después (B) de la inyección intravítrea de plasmina autóloga (paciente 1). Sin cambios anatómicos ni visuales. 

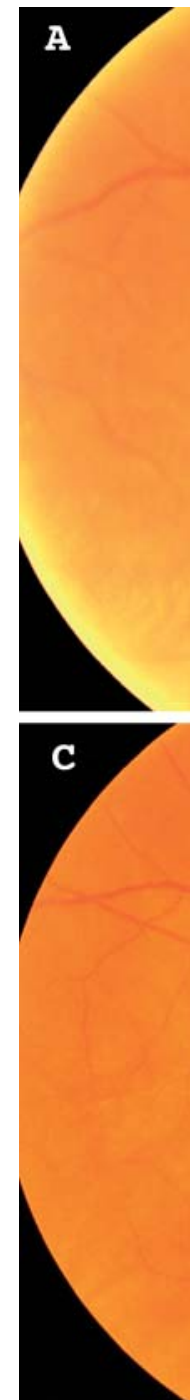
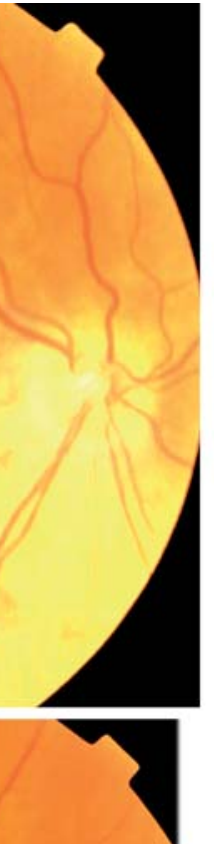

\section{D.}

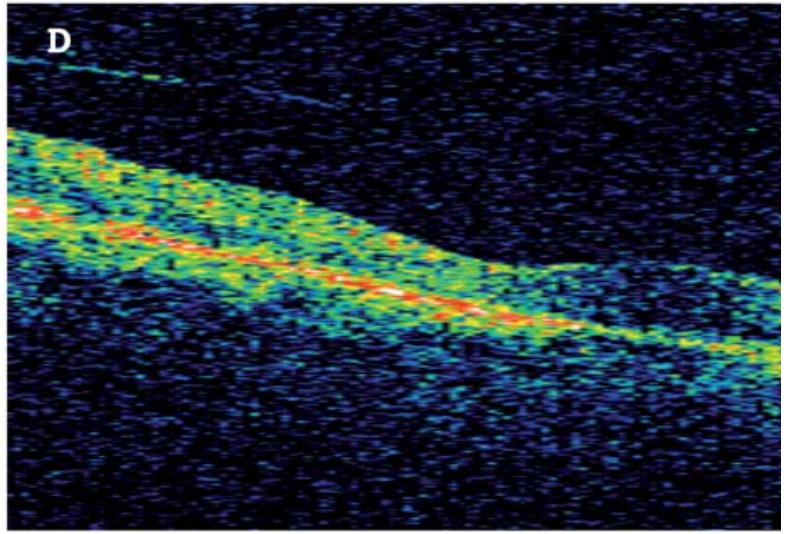

Fig. 3: Síndrome de tracción vitreomacular (STVM) antes ( $A$ y B) y después ( $C$ y D ) de la inyección de plasmina intravítrea. Obsérvese el despegamiento de la tracción del área macular oftalmoscópicamente y la normalización de la estructura anatómica macular en la tomografía de coherencia óptica (paciente 5). La agudeza visual mejoró desde 0,2 a 0,8 .

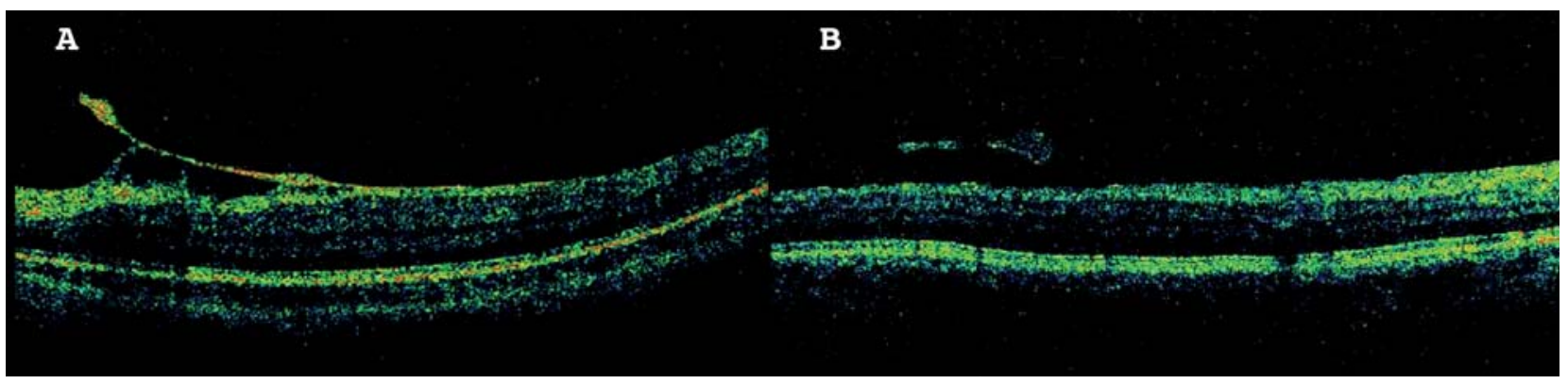

Fig. 4: Síndrome de tracción vitreomacular (STVM) antes (A) y después ( B) de la inyección de plasmina intravítrea. Obsérvese la normalización de la estructura anatómica retiniana en la tomografia de coherencia óptica (paciente 6). La agudeza visual mejoró desde 0,4 a 0,9. 
abre la posibilidad a empezar a valorarse y considerarse una alternativa sencilla y segura previa a intentar maniobras quirúrgicas como el pelado de dichas membranas. Dicha eficacia ha resultado negativa en la serie inicial de casos estudiada con membrana epirretiniana (MEM). Hasta la actualidad la inyección intravítrea de plasmina se había utilizado siempre inmediatamente antes de la vitrectomía para facilitar el manejo intraoperatorio (16,27-34). Recientemente la inyección intravítrea de plasmina autóloga como tratamiento único -sin vitrectomía quirúrgica asociada- ha demostrado ser parcialmente eficaz en el edema macular difuso diabético (35). La eficacia de la inyección aislada de plasmina en conseguir la separación farmacológica del cortex posterior vítreo de la retina, junto con su efecto vitreolítico, conocida como vitrectomía enzimática y vitreolisis farmacológica $(16,17,41)$, abre la posibilidad terapéutica no sólo del edema macular diabético y del STVM, sino de otros edemas maculares (oclusiones venosas, uveítis, postquirúrgico, etc.) y otras patologías donde las tracciónes vitreorretininas estén claramente implicadas en su patogenia (síndrome de tracción macular miópica, agujeros maculares, síndrome de edema macular cistoideo traccional, etc.) (42), sin los riesgos yatrogénicos asociados de la cirugía vítrea (cataratas $>80 \%$, desgarros -20\%-, desprendimientos de retina, hemorragia vítrea, etc.) (43-45).

Aunque podría no existir una correlación directa entre mejoría de la agudeza visual y el despegamiento de la membrana, ésta sí ha ocurrido en nuestros casos; los pacientes en los que la plasmina despegó la membrana de la retina en el área macular -siempre casos de STVM- fueron los que incrementaron su visión con desaparición de la metamorfopsia y normalización de los parámetros cualitativos visuales (nitidez, luminosidad, contraste y colorimetría).

Debemos recordar que la inyección de plasmina se utilizó como primera opción terapéutica antes que intentar su tratamiento mediante pelado quirúrgico de la membrana epirretiniana macular (MEM) y de la membrana del STVM. La mejoría visual ocurrida exclusivamente en los pacientes con STVM y no en los casos con MEM, es percibida por el paciente muy precozmente (2-3 días), manteniéndose estable durante todo el seguimiento. Este estudio es piloto y por tanto sus resultados no generalizables. Estudios con mayor número de casos y seguimientos a largo plazo son necesarios para establecer su eficacia como monoterapia, y la posible eficacia de reinyecciones para consolidar su eficacia en los casos fracasados de MEM. No es teóricamente imposible que reinyecciones de plasmina sean capaces de conseguir el despegamiento de la MEM en los casos en que no lo logró la primera inyección, pero depende de futuros estudios establecer el número y la frecuencia con que deben realizarse dichas inyecciones antes de considerar la técnica fracasada y proceder a la cirugía.

La gran diferencia de eficacia de la plasmina autóloga inyectada intravítrea entre los pacientes con MEM y STVM es un un punto importante de reflexión. En primer lugar, la tomografía de coherencia óptica (OCT) permite más claramente diferenciar ambos síndromes que por biomicroscopía pueden fácilmente ser confundidos, incrementando la identificación de estas patologías frente a la biomicroscopía en un $10 \%$ en los casos de MEM y hasta un $300 \%$ en el STVM (46-48). Observando las figuras 1, 2, 3 y 4 puede observarse la diferencia tomográfica entre ambas patologías. En segundo lugar, la ausencia de eficacia de la plasmina en las MEM podría explicarse porque en ellas se asocia en más del $95 \%$ un desprendimiento posterior del vítreo previo (49-52). Existen casos de MEM con vítreo adherido exclusivamente en jóvenes (53) -ninguno de ellos incluido en nuestra serie-, en los que la eficacia de la vitrectomía quirúrgica ha resultado muy superior a la realizada en pacientes con DPV (53). Tal vez sea este subgrupo de MEM -con hialoides adherida en sujetos jóvenes- el único suceptible de ser tratado con plasmina intravítrea. En tercer lugar, debemos recordar que las nuevas generaciones de OCT tridimensionales de alta definición van haciendo progresivamente más compleja e indiferenciables ambos síndromes (MEM versus STVM). Así, Koizumi y cols (55) observaron la coexistencia en 10 de 12 casos de STVM de una MEM subyacente. Esta observación tomográfica de superposición clínica de ambos síndromes ha sido corroborado histológicamente (56-60), donde las membranas de ambas patologías resultan ser similares con la única diferencia de asociar fragmentos de la membrana limitante interna en las MEM. Por último en cuarto lugar, en los casos de STVM, donde la plasmina intravítrea ha resultado eficaz, esta opción se perfila como una opción terapéutica inicial mínimamente invasiva, sabiendo que es imprevisible la evolución del mismo sin tratamiento, evolucionando algunos casos de manera espontánea hacia el despegamiento completo y resolución entre 
1 y 6 meses desde el comienzo de los síntomas (1114), y en otros hacia la progresión a un agujero macular completo (61).

La eficacia y toxicidad de la plasmina autóloga es dosis-dependiente y tiempo-dependiente. La inyección intravítrea de 0,4 UI de plasmina ha demostrado en estudios previos ser suficiente para separar el cortex vítreo posterior de la limitante interna, sin toxicidad conocida hasta concentraciones de 3-4 UI (62-64). La cantidad de plasmina autóloga obtenida e inyectada por el método empleado en este estudio es de media de 0,26 UI $(15,38)$, pero ha demostrado ser suficiente para ser eficaz en todos los casos tratados de síndrome de tracción vitreomacular (STVM), y es hipotético pero no imposible que concentraciones mayores hubieran conseguido ser eficaces en las membranas epirretinianas (MEM). Gandorfer y cols. $(26,63,65)$ encontraron una correlación directa entre tiempo de exposición de la plasmina y grado de separación vítreorretiniana. Estudios in vitro han comprobado diferentes tiempos de exposición a la plasmina para las diferentes moléculas fundamentales en la adhesión vitreoretiniana: la laminina está completamente degradada en 15 minutos, mientras que la fibronectina sigue degradándose bajo la acción de la plasmina durante 60 minutos, no siendo detectable efecto alguno más allá de las 24 horas (65-67). Partiendo del principio de que la plasmina autóloga residual en los estudios previos ha sido siempre evacuada del vítreo en la maniobra quirúrgica inmediatamente posterior a la inyección realizada, creemos que a la vista de nuestros resultados más que como coadyuvante quirúrgico, la plasmina intravítrea podría utilizarse como alternativa previa a la vitrectomía quirúrgica en los casos de STVM. El tiempo de exposición de la superficie vitreorretiniana a la plasmina autóloga ha demostrado en el presente estudio ser suficiente y sin toxicidad. Además, el cambio de la estreptoquinasa por uroquinasa como activador del plasminógeno a plasmina $(29,30,37,38)$, tiene la ventaja añadida de un menor potencial de toxicidad intraocular además de evitar el enturbiamiento inicial del vítreo que ocurría en la mayoría de casos cuando se preparaba la plasmina con estreptoquinasa $(16,35,36)$. No se ha observado en ningún caso complicación alguna como endoftalmitis, hemorragia vítrea, uveítis, desprendimiento de retina, aumento de la presión intraocular o progresión de catarata.

Uno de los principales problemas que limitaban el uso de plasmina autóloga ha sido siempre su sofisticado sistema de preparación, largo, costoso y complejo, requiriendo equipamientos especializados sólo accesibles en determinadas unidades hematológicas. La ventaja de la técnica simplificada de preparación de plasmina autóloga es la posibilidad de ser preparada en el propio quirófano de Oftalmología unos minutos antes de su uso, y de manera rápida, fácil y relativamente económica $(16,37)$. La futura comercialización de la microplasmina (Trombogenics, Irlanda) permitirá una utilización más universal de esta técnica (69) (Stalman P. MIVI-2T Trial. A randomized, double-masked clinical trial of microplasmin intravitreal injection for nonsurgical treatment of vitreomacular traction. Indian Wells, California: American Society of Retina Specialist; 2007) (Brown D. MIVI III Trial. Boston: Retina Society Annual Meeting; 2007).

En conclusión, la vitrectomía enzimática y farmacológica realizada únicamente con la inyección intravítrea de plasmina autóloga ha demostrado ser eficaz y segura en reducir y eliminar la tracción macular, y mejorar la agudeza visual en los casos de síndrome de tracción vitreomacular (STVM), evitando la vitrectomía quirúrgica, y en ningún caso de membrana epirretiniana macular (MEM) de la serie estudiada. Futuros estudios deberán matizar la dosis intravítrea de plasmina más idónea y eficaz en el síndrome de tracción vitreomacular, la posibilidad de incrementar su eficacia en los casos de MEM inicialmente resistentes con sucesivas reinyecciones y/o a mayores dosis, así como su papel como facilitador de su despegamiento durante la vitrectomía quirúrgica posterior.

\section{BIBLIOGRAFÍA}

1. Koerner F, Garweg J. Diseases of vítreo-macular interface. Klin Monatsbl Augenheilkd 1999; 214: 305-310.

2. Clarkson JG, Green WR, Massot D. A histopathologic review of 168 cases of preretinal membrane. Am J Ophthalmol 1977; 84: 1-17.

3. Gailit J, Clark RAF. Wound repair in the context of extracellular matrix. Curr Opin Cell Biol 1994; 6: 717-725.

4. Wiedemann P, Heimann K. Proliferative vitreoretinopathy. Curr Opin Ophthalmol 1992; 3: 357-365.

5. Messmer EM, Heidenkummer HP, Kampik A. Ultraestructure of epiretinal membranes associated with macular holes. Graefes Arch Clin Exp Ophthalmol 1998; 236: 248254.

6. Immonen I, Vaheri A, Tommila P, Siren V. Plasminogen activation in epiretinal membranas. Graefe's Arch Clin Exp Ophthalmol 1996; 234: 664-669. 
7. Greven CM, Slusher MM, Weaver RG. Epiretinal membrane release and posterior vitreous detachment. Ophthalmology 1988; 95: 902-905.

8. Garay-Aramburu G, Larrauri-Arana A. Resolución espontánea de membrana epirretiniana idiopática en un paciente joven. Arch Soc Esp Oftalmol 2005; 80: 741-743.

9. Hikichi T, Yoshida A, Trempe CL. Course of vitreomacular traction syndrome. Am J Ophthalmol 1995; 119: 55-61.

10. Sarup V, Wald KJ, Gross NE, Athanikar AN, Siringo F. Spontaneous resolution of unilateral vitreomacular traction. Ann Ophthalmol 2006; 38: 347-349.

11. Levy J, Klemperer I, Belfair N, Rogozin A, Liftshitz T. Rapid spontaneous resolution of vitreomacular traction syndrome documented by optical coherence tomography. Int Ophthalmol 2004; 25: 247-251.

12. Rodriguez, A, Infante R, Rodriguez FJ, Valencia M. Spontaneous separation in idiopathic vitreomacular traction syndrome associated with contralateral full-thickness macular hole. Eur J Ophthalmol 2006; 16: 733-740.

13. Carpineto P, Ciancaglini M, Aharrh-Gnama A, Agnifili L, Mastropasqua L. Optical coherence tomography and retinal thickness. Analyzer features of spontaneous resolution of vitreomacular traction syndrome. A case report. Eur J Ophthalmol 2004; 14: 67-70.

14. Maguluri S, Recchia FM. Resolution of tractional macular edema following spontaneous detachment of residual vitreous cortex in a vitrectomized eye. Ophthalmic Surg Laser 2008; 39: 63-64.

15. Rizzo S, Pellegrini G, Benocci F, Belting C, Baicchi M. Autologous plasmin for pharmacologic vitreolysis prepared 1 hour before surgery. Retina 2006; 26: 792-796.

16. Tanaka $M$, Qui H. Pharmacological vitrectomy. Semin Ophthalmol 2000; 15: 51-61.

17. Alió J, Sánchez A, Ludeña S, García S, Bergés J, Batlle J. Vitreolisis enzimática: estudio experimental de un nuevo método no mecánico de vitrectomia. Arch Soc Esp Oftal 1987; 53: 349-360.

18. Trese MT. Enzymatic-assisted vitrectomy. Eye 2002; 16: 365-368.

19. Sebag J. Molecular biology of pharmacologic vitreolysis. Trans Am Ophthalmol Soc 2005; 103: 473-493.

20. Verstraten TC, Chapman C, Hartzer M, Winkler BS, Trese MT, Williams GA. Pharmacological induction of posterior vitreous detachment in the rabbit. Arch Ophthalmol 1993; 111: 849-854.

21. Kohno T, Sorgente N, Patterson R, Ryan SJ. Fibronectin and laminin distribution in bovine eye. Jpn J Ophthalmol 1983; 27: 496-505.

22. Kohno T, Sorgente N, Ishibashi T, Goodnight R, Ryan SJ. Immunofluorescent studies of fibronectin and laminin in the human eye. Invest Ophthalmol Vis Sci 1987; 28: 506-514.

23. Liotta LA, Goldfarb RH, Terranova VP. Cleavage of laminin by thrombin and plasmin: alpha thrombin selectively cleaves the beta chain of laminin. Thromb Res 1981; 21: 663-673.

24. Hikichi T, Yanagiya N, Kado M, Akiba J, Yoshida A. Posterior vitreous detachment induced by injection of plasmin and sulfur hexafluoride in the rabbit vitreous. Retina 1999; 19: 55-58.

25. Li X, Shi X, Fan J. Posterior vitreous detachment with plasmin in the isolated human eye. Graefes Arch Clin Exp Ophthalmol 2002; 240: 56-62.
26. Gandofer A, Putz E, Welge-Lupben U, Grüterich M, Ulbig $M$, Kampik A. Ultrastructure of the vitreoretinal interface following plasmin assisted vitrectomy. Br J Ophthalmol 2001; 85: 6-10.

27. Asami T, Terasaki H, Kachi S, Nakamura M, Yamamura K, Nabeshima T, et al. Ultrastructure of internal limiting membrane removed during plasmin assisted vitrectomy from eyes with diabetic macular edema. Ophthalmology 2004; 111: 231-237.

28. Trese MT, Williams GA, Hartzer MK. A new approach to stage 3 macular hole. Ophthalmology 2000; 107: 1607-1611.

29. Sakuma T, Tanaka M, Inoue J, Mizota A, Souri M, Ichinose A. Use of autologous plasmin during vitrectomy for diabetic maculopathy. Eur J Ophthalmol 2006; 16: 138-140.

30. Sakuma T, Takana M, Inoue J, Mizota A, Sauri M, Achinose A. Efficacy of autologous plasmin for idiopathic macular hole surgery. Eur J Ophthalmol 2005; 15: 787-794.

31. Azzolini C, D'Angelo A, Maestranzi G, Codenotti M, Della Valle P, Prati M, et al. Intrasurgical plasmin in diabetic macular edema. Am J Ophthalmol 2004; 138: 560-568.

32. Williams JG, Trese MT, Williams GA, Hartzer MK. Autologous plasmin enzyme in the surgical management of diabetic retinopathy. Ophthalmology 2001; 108: 1902-1905.

33. Margherio AR, Margherio RR, Hartzer $M$, Trese MT, Williams GA, Ferrone PJ. Plasmin enzyme-assisted vitrectomy in traumatic pediatric macular holes. Ophthalmology 1998; 105: 1617-1620.

34. Tsukahara Y, Honda S. Autologous plasmin-assisted vitrectomy for stage 5 retinopathy of prematurity. Am J Ophthalmol 2007; 144: 139-141.

35. Diaz-Llopis M, Udaondo P, García-Delpech S, Salom D, Cervera E, Quijada A. Vitrectomía enzimática por inyección intravítrea de plasmina autóloga como tratamiento inicial del edema macular diabético difuso. Arch Soc Esp Oftalmol 2008; 83: 77-84.

36. Udaondo P, Diaz-Llopis M, García-Delpech S, Cervera E, Salom D. Plasmina autóloga para inyección intravítrea sin vitrectomía asociada: método simplificado de preparación con streptokinasa. Arch Soc Esp Oftalmol 2008; 83: 153-154.

37. Diaz-Llopis M, Udaondo P, Salom D, García-Delpech $S$, Romero FJ. Plasmina autóloga intravítrea sin asociar vitrectomía. Vitreolisis farmacológica, método perfeccionado con uroquinasa. Arch Soc Esp Oftalmol 2008; 83: 291-292.

38. Unal M, Peyman GA. The efficacy of plasminogen-urokinase combination in inducing posterior vitreous detachment. Retina 2000; 20: 69-75.

39. Men G, Peyman GA, Genaidy M, Kuo PC, Ghahramani $F$, Blake DA, Bezerra Y, Naaman G, Figueiredo E. The role of recombinant lysine-plasminogen and recombinant urokinase and sulfur hexafluoride combination in inducing posterior vitreous detachment. Retina 2004; 24: 199-209.

40. Sakuma T, Tanaka M, Saouri M, Ichinose A. Preparation of high-purity and safe autologous plasmin and its clinical application. Nippon Ganka Gakkai Zasshi 2003; 107: 709-718.

41. Treese MT. Enzymatic vitreous surgery. Semin Ophthalmol 2000; 15: 161-121.

42. Johnson MW. Tractional cystoid macular edema: a subtle variant of the vitreomacular traction syndrome. Am J Ophthalmol 2005; 140: 184-192. 
43. Melberg NS, Williams DF, Balles MW, Jaffe GJ, Meredith TA, Sneed SR, Westrich DJ. Vitrectomy for vitreomacular traction syndrome with macular detachment. Retina 1995, 15: 192-197.

44. Pournaras CJ, Kapetanios AD, Donati G. Vitrectomy for traction macular edema. Doc Ophthalmol 1999; 97: 439447.

45. McDonald HR, Johnson RN, Schatz H. Surgical results in the vitreomacular traction syndrome. Ophthalmology 1994; 101: 1397-1402.

46. Yamada N, Kishi S. Tomographic features and surgical outcomes of vitreomacular traction syndrome. Am J Ophthalmol 2005; 139: 112-117.

47. Do DV, Cho M, Nguyen QD, Shah SM, Handa JT, Campochiaro PA, et al. Impact of optical coherence tomography on surgical decision making for epiretinal membranes and vitreomacular traction. Retina 2007; 27: 552-556.

48. Mirza RG, Johnson MW, Jampol LM. Optical coherence tomography use in evaluation of the vitreoretinal interface: a review. Surv Ophthalmol 2007; 52: 397-421.

49. Wiznia RA. Natural history of idiopathic preretinal macular fibrosis. Ann Ophthalmol 1982; 14: 876-878.

50. Wise GN. Relationship of idiopathic preretinal macular fibrosis to posterior vitreous detachment. Am J Ophthalmol 1975; 79: 358-362.

51. Hirokawa H, Jalkh AE, Takahashi M, Takahashi M, Trempe $C L$, Schepens $C L$. Role of the vitreous in idiopathic preretinal macular fibrosis. Am J Ophthalmol 1986; 101: 166-169.

52. Wiznia RA. Posterior vitreous detachment and idiopathic preretinal macular gliosis. Am J Ophthalmol 1986; 102: 196-198.

53. Fang $X$, Chen Z, Weng Y, Shu Z, Ni H, Jiang J, et al. Surgical outcome after removal of idiopathic epiretinal membrane in young patients. Eye 2008; 22: 1430-1435.

54. Heilskov TW, Massicotte SJ, Folk JC. Epiretinal macular membranes in eyes with attached posterior cortical vitreous. Retina 1996; 16: 279-284.

55. Koizumi H, Spaide RF, Fisher YL, Freund KB, Klancnik $J M$, Yanuzzi LA. Three-dimensional evaluation of vitreomacular traction and epiretinal membrane using spectraldomain optical coherence tomography. Am J Ophthalmol 2008; 145: 509-517.
56. Simiddy WE, Maguire AM, Green WR. Idiopathic epiretinal membranes. Ultraestructural characteristics and clinicopathologic correlation. Ophthalmology 1989; 96: 811-820.

57. Smiddy WE, Green WR, Michels RG, De la Cruz Z. Ultrastructural studies of vitreomacular traction syndrome. Am J Ophthalmol 1989; 107: 177-185.

58. Shinoda K, Hirakata A, Hida T, Yamaguchi Y, Fukuda M, Maekawa S, et al. Ultrastructural and immunohistochemical findings in five patients with vitreomacular traction syndrome. Retina 2000; 20: 289-293.

59. Kampik A, Green WR, Michaels RG, Nase PK. Ultrastructural features of progressive idiopathic epiretinal membrane removed by vitreous surgery. Am J Ophthalmol 1980; 90: 797-809.

60. Gandorfer A, Rohleder M, Kampik A. Epiretinal pathology of vitreomacular traction syndrome. $\mathrm{Br} J$ Ophthalmol 2002; 86: 902-909.

61. Lee MW, Cheng Bobby CL, Ang CL. Optical coherence tomography evidence of rapid progression from vitreo macular traction to full thickness macular hole. Clin Exp Ophthalmol 2007; 35: 379-380.

62. Wang $F$, Wang Z, Sun X, Wang F, Xu X, Zhang X. Safety and efficacy of dispase and plasmin in pharmacologic vitreolysis. Invest Ophthalmol Vis Sci 2004; 45: 3286-3290.

63. Gandorfer A. Pharmacological vitreolysis. Dev Ophthalmol 2007; 39: 149-156.

64. Hermel M, Mahgoub M, Youssef T, Azrak MI, Raza H, Alldredge $C$, et al. Safety profile of the intravitreal streptokinase-plasmin complex as an adjuvant to vitrectomy in the rabbit. Graefes Arch Clin Exp Ophthalmol 2006; 244: 996-1002.

65. Gandorfer A, Ulbig M, Kampic A. Plasmin-assisted vitrectomy eliminates cortical vitreous remmants. Eye 2002; 16: 95-97.

66. Uemura A, Nakamura M, Kachi S, Nishizawa Y, Asami T, Miyake $Y$, et al. Effect of plasmin on laminin and fibronectin during plasmin-assisted vitrectomy. Arch Ophthalmol 2005; 123: 209-213.

67. Gandorfer A, Priglinger S, Schebitz K. Vitreoretinal morphology of plasmin-treated human eyes. Am J Ophthalmol 2002; 133: 156-159.

68. Gandorfer A. Enzymatic vitreous disruption. Eye, 2008, 22: 1273-1277. 